\title{
vcftoolz: a Python package for comparing and evaluating Variant Call Format files.
}

\section{Steve Davis ${ }^{1}$}

1 U.S. Food and Drug Administration

DOI: $10.21105 /$ joss.01144

\section{Software}

- Review ¿

- Repository ¿

- Archive c兀

Submitted: 27 November 2018 Published: 26 March 2019

\section{License}

Authors of papers retain copyright and release the work under a Creative Commons Attribution 4.0 International License (CC-BY).

\section{Summary and Need Statement}

The analysis of next-generation sequence data often involves variant calling - the process of identifying differences between genomes. The standard output file format of variant callers is the Variant Call Format (VCF) (https://github.com/samtools/hts-specs).

Researchers need to view and compare VCF files when comparing the behavior of different variant calling algorithms (and even the same algorithm with different parameters). Additionally, the performance of a variant calling algorithm can be evaluated by comparing against a known truth VCF dataset.

Here, we present vcftoolz, software to facilitate comparing and evaluating the variant calls in VCF files. The core functionality of vcftoolz is the capability to compare two or more VCF files, producing a report, Venn Diagrams, and a spreadsheet identifying the concordance between the VCF files. The artifacts produced by vcftoolz are not available from other tools.

The vcftoolz software is designed to work with bacterial variant files. It has been tested in a 3-way comparison of VCF files, with each file having 200 samples and 500 snps per sample for a total of 100,000 variants in each file.

\section{Related Research}

The vcftoolz software is being used as part of an ongoing effort to compare and evaluate the variant callers used by multiple government agencies involved in the analysis of pathogenic organisms of interest to food safety. In this effort, we use multiple variant callers to construct VCF files from food-borne pathogens. The vcftoolz software identifies the concordance between the VCF files produced by the alternative variant callers and facilitates algorithm improvements.

\section{Prior Related Work}

The RTG Tools package (Cleary et al., 2015) has advanced capabilities to compare VCF files containing complex variants, but does not support VCF files with multiple samples per file.

The BCFtools (Clarke et al., n.d.) package has the capability to create intersections, unions and complements of VCF files, as well as other useful tools for working with VCF files. 
The VCFtools (Danecek et al., 2011) package has the capability to calculate differences between VCF files, among other functions.

\section{Links}

Documentation: https://vcftoolz.readthedocs.io/en/latest/readme.html

Source Code: https://github.com/CFSAN-Biostatistics/vcftoolz

PyPI Distribution: https://pypi.python.org/pypi/vcftoolz

\section{References}

Clarke, N., Collier, T., Danecek, P., Herrero, J., Kretzschmar, W., Li, H., McCarthy, S., et al. (n.d.). BCFtools. Retrieved November 26, 2018, from https://github.com/ samtools/bcftools

Cleary, J. G., Braithwaite, R., Gaastra, K., Hilbush, B. S., Inglis, S., Irvine, S. A., Jackson, A., et al. (2015). Comparing variant call files for performance benchmarking of nextgeneration sequencing variant calling pipelines. BioRxiv, 023754. doi:10.1101/023754

Danecek, P., Auton, A., Abecasis, G., Albers, C. A., Banks, E., DePristo, M. A., Handsaker, R. E., et al. (2011). The variant call format and vcftools. Bioinformatics, 27(15), 2156-2158. doi:10.1093/bioinformatics/btr330 